\title{
Development of an improved Pseudoalteromonas haloplanktis TACI 25 strain for recombinant protein secretion at low temperature
}

\author{
Ermenegilda Parrilli1,2, Daniela De Vizio ${ }^{1}$, Claudia Cirulli1,3 and \\ Maria Luisa Tutino*1,2
}

\begin{abstract}
Address: ${ }^{1}$ Dipartimento di Chimica Organica e Biochimica, Università degli studi di Napoli Federico II - Complesso Universitario M.S. Angelo via Cinthia 4, 80126, Napoli Italia, ${ }^{2}$ Facoltà di Scienze Biotecnologiche, Università degli studi di Napoli Federico II, Napoli Italia and ${ }^{3}$ Dipartimento di Biotecnologie e Bioscienze dell'Università Milano-Bicocca, Piazza della scienza 2, 20126, Milano Italia
\end{abstract}

Email: Ermenegilda Parrilli - erparril@unina.it; Daniela De Vizio - dana.devizio@googlemail.com; Claudia Cirulli - cirulli@unina.it; Maria Luisa Tutino* - tutino@unina.it

* Corresponding author

Published: 7 February 2008

Microbial Cell Factories 2008, 7:2 doi:10.1 186/1475-2859-7-2
Received: 5 December 2007

Accepted: 7 February 2008

This article is available from: http://www.microbialcellfactories.com/content/7/1/2

(C) 2008 Parrilli et al; licensee BioMed Central Ltd.

This is an Open Access article distributed under the terms of the Creative Commons Attribution License (http://creativecommons.org/licenses/by/2.0), which permits unrestricted use, distribution, and reproduction in any medium, provided the original work is properly cited.

\begin{abstract}
Background: In a previous paper, we reported the accomplishment of a cold gene-expression system for the recombinant secretion of heterologous proteins in Pseudoalteromonas haloplanktis TACI25. This system makes use of the psychrophilic $\alpha$-amylase from P. haloplanktis TAB23 as secretion carrier, and allows an effective extra-cellular addressing of recombinant proteins. However, Pseudoalteromonales are reported to secrete a wide range of extra-cellular proteases. This feature works against the efficiency of the cold-adapted secretion system, because of the proteolytic degradation of recombinant products. The aim of this study is the construction of a P. haloplanktis TACI 25 mutant strain with reduced extra-cellular proteolytic activity.

Results: P. haloplanktis TACI 25 culture medium resulted to contain multiple and heterogeneous proteases. Since the annotation of the Antarctic bacterium genome highlighted the presence of only one canonical secretion machinery, namely the Type II secretion pathway (T2SS), we have inactivated this secretion system by a gene insertion strategy. A mutant strain of $P$. haloplanktis TACI 25 in which the gspE gene was knocked-out, actually displayed a remarkable reduction of the extra-cellular protease secretion. Quite interestingly this strain still retained the ability to secrete the psychrophilic amylase as efficiently as the wild type. Moreover, the decrease in extra-cellular proteolytic activity resulted in a substantial improvement in the stability of the secreted amylase- $\beta$-lactamase chimera.

Conclusion: Here we report a cell engineering approach to the construction of a P. haloplanktis TACI 25 strain with reduced extra-cellular protease activity. The improved strain is able to secrete the psychrophilic $\alpha$-amylase (the carrier of our recombinant secretion system), while it displays a significant reduction of protease content in the culture medium. These features make the gspE mutant an improved host with a remarkable biotechnological potential in recombinant protein secretion at low temperature. Moreover this work demonstrates that $P$. haloplanktis TACI 25 is a versatile psychrophilic host for recombinant protein production since it can be easily improved by a directed engineering approach. To the best of our knowledge, this is the first described example of a strain improvement strategy applied to an Antarctic bacterium.
\end{abstract}




\section{Background}

Protein secretion into the extra-cellular environment is one of most desirable strategy to allow a rapid and not expensive recovery of recombinant proteins. Secretion to the culture medium has several advantages over intracellular recombinant protein production. These advantages include simplified downstream processing, enhanced biological activity, higher product stability and solubility, and N-terminal authenticity of the expressed peptide [1$3]$. If the product is secreted to the culture medium, cell disruption is not required for recovery. As bacteria, usually, do not secrete amounts of proteins higher then they have in the intracellular space, recovery of a recombinant gene product can be greatly simplified by a secretion strategy that minimises contamination from host proteins. Additionally, secretion can provide a method to guarantee the N-terminal authenticity of the expressed polypeptide because it often involves the cleavage of a signal sequence [4], thus avoiding the presence of an unwanted initial methionine on a protein that does not normally contain it. This extra methionine can reduce the biological activity and stability of the product [5] or even elicit an immunogenic response in the case of therapeutic proteins.

In a previous paper [6], we reported the realization of a "cold" recombinant secretion system in the Antarctic Gram-negative bacterium P. haloplanktis TAC125. This system efficiently conjugates the obvious advantages of extracellular protein targeting with the positive effect of low temperature on the recombinant product solubility. Indeed, low expression temperature can facilitate the correct folding of "difficult" products $[7,8]$ and the use of $P$. haloplanktis TAC125 as expression system [9] allowed the efficient production of some "intractable" proteins in soluble and active form at temperature as low as $4^{\circ} \mathrm{C}[10$ $12]$.

The cold-adapted secretion system [6] makes use of the psychrophilic $\alpha$-amylase from $P$. haloplanktis TAB23 $[13,14]$ as secretion carrier. Three chimerical proteins, made of the psychrophilic $\alpha$-amylase fused to an intra-cellular protein, were translocated in the extra-cellular medium with a secretion yield always higher than $80 \%$. The system also allowed the correct disulphide bond formation of chimera components, secreting a fully active passenger [6]. However, our previous results addressed to a potential limit of this newly set up technology: host extra-cellular medium may contain proteolytic activities which can affect the quality of heterologous products [6]. This feature could hamper the applicability of the coldadapted secretion system, due to the likely recombinant product degradation.

To overcome this host limit, two possible approaches can be pursued thanks to the availability of $P$. haloplanktis
TAC125 genome sequencing and annotation [15]: i) the gene disruption of each $P$. haloplanktis TAC125 gene encoding extra-cellular proteases; or ii) the inactivation of the secretion machinery responsible for the proteases extra-cellular targeting.

The in silico analysis of $P$. haloplanktis TAC125 genome demonstrated that the bacterium possesses only one canonical secretion system, a putative Type II secretion machinery (T2SS) also called General Secretory Pathway (GSP), homologous to GSPs already described in many other Gram-negative bacteria [16]. Since experimental evidences suggested that in $P$. haloplanktis TAC125 the secretion of the cold $\alpha$-amylase depends on a still uncharacterized pathway (unpublished results from this laboratory), the inactivation of the T2SS machinery seemed a reasonable strategy to develop a $P$. haloplanktis TAC125 mutant strain with reduced extra-cellular proteolytic activity.

In this paper we report the set-up of an integrative plasmid and its use for the construction of a $P$. haloplanktis TAC125 strain in which the $g s p E$ gene $[17,18]$ was knocked-out. This mutation resulted in the inactivation of the psychrophilic T2SS system. The P. haloplanktis TAC125 gspE mutant displayed a remarkable reduction of the extra-cellular protease secretion, still maintaining its ability to secrete the psychrophilic amylase (the secretion carrier of our recombinant system) as efficiently as the wild type. These features make the $P$. haloplanktis TAC125 gspE mutant strain an improved host with a remarkable biotechnological potential in recombinant protein secretion at low temperature.

\section{Results \\ Pseudoalteromonas haloplanktis TACI 25 growth medium contains several secreted proteases}

Wild type $P$. haloplanktis TAC125 cells were grown in TYP medium at $4^{\circ} \mathrm{C}$ and culture medium samples were withdrawn at different growth phases (at 24, and 32 hours of incubation corresponding to early and medium exponential phase). Concentrated culture supernatants were analyzed for proteolytic activities using Gelatine-SDS-PAGE (10\% acrylamide, $\mathrm{w} / \mathrm{v}$ ) as described in Materials and Methods. As shown in Figure 1A, the wild type P. haloplanktis TAC125 culture supernatants contain a wide range of proteolytic activities, which display an apparent molecular weight ranging between 120 and $33 \mathrm{kDa}$.

A protease inhibition assay was performed by treating the 24 hours extra-cellular protein sample with $10 \mathrm{mM}$ PMSF (a serine protease inhibitor) for 20 hours at $15^{\circ} \mathrm{C}, 10 \mathrm{mM}$ EDTA (a metalloprotease inhibitor) for 20 hours at $15^{\circ} \mathrm{C}$, and with the combination of the two inhibitors. The differentially treated samples were then subjected to gelatine 


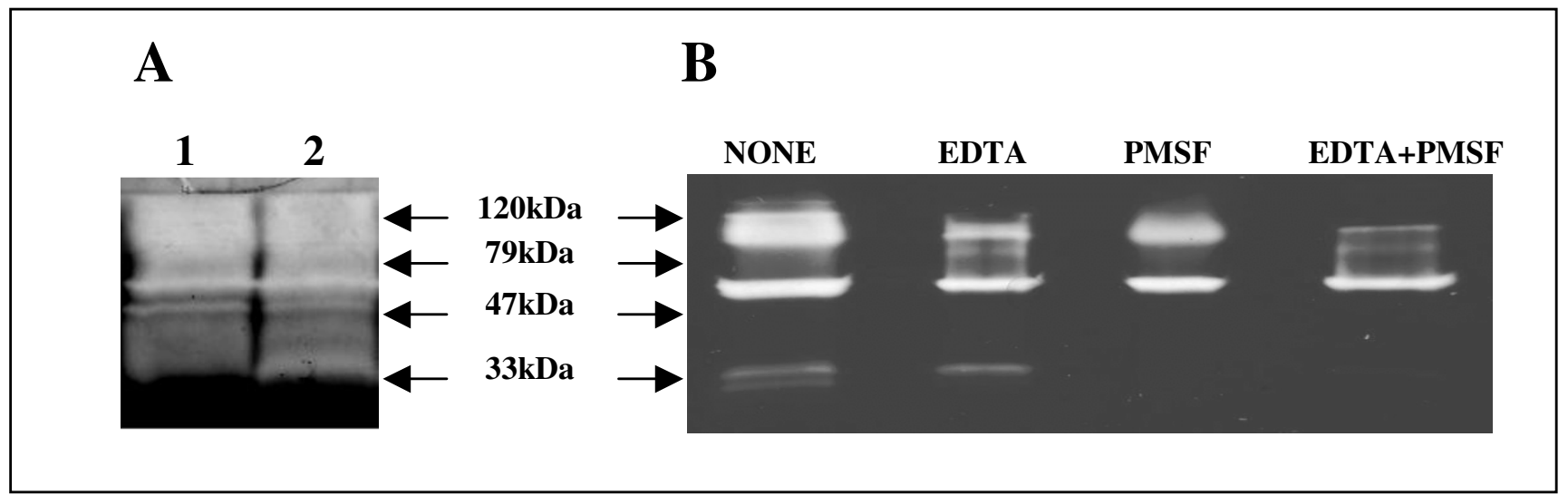

\section{gspE mutant}

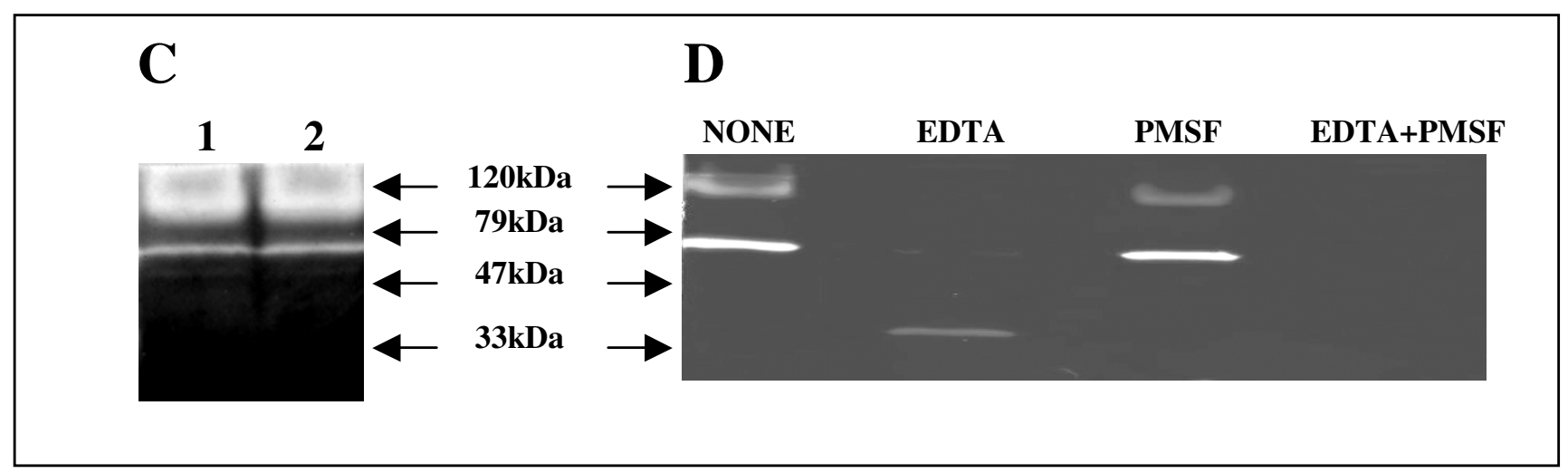

Figure I

In gel analysis of extra-cellular proteolytic activities from culture supernatants of $P$. haloplanktis TACI25 wild type and gspE mutant strain. Panel A: Zymography of $P$. haloplanktis TACI 25 wild type culture supernatants collected at early $(24 \mathrm{~h})$ (lane I), and middle (32 h) (lane 2) exponential phase. In this experiment the zymographic developing time was I 8 $\mathrm{h}$, a condition that assures the detection of all proteases contained in the sample. Panel B: Protease zymography of a $P$. haloplanktis TACI 25 wild type culture supernatant, collected at $24 \mathrm{~h}$, untreated (NONE) and treated with protease inhibitors ( 10 $\mathrm{mM}$ EDTA, $10 \mathrm{mM}$ PMSF, and the combination of the two inhibitors both at $10 \mathrm{mM}$ final concentration). In this experiment a zymographic developing time of $12 \mathrm{~h}$ was chosen, this condition allows a clearer visualization and comparison of the proteases contained in the different samples. Panel C: Protease zymography of $P$. haloplanktis TACI 25 gspE mutant culture supernatants collected at early $(24 \mathrm{~h}$ ) (lane I), and middle (32 h) (lane 2) exponential phase, the zymographic developing time was I8 h. Panel D: Protease zymography of a P. haloplanktis TACI 25 gspE mutant culture supernatant, collected at 24 h, untreated (NONE) and treated with protease inhibitors, the zymographic developing time was $12 \mathrm{~h}$ ).

zymography, and the results are shown in Figure 1B. A comparative evaluation of the results demonstrates that the wild type $P$. haloplanktis TAC125 culture supernatant contains extra-cellular proteases inhibited either by PMSF or by EDTA, and proteolytic activities which appear to be resistant to both the inhibitors (Figure $1 \mathrm{~B}$ lane EDTA+PMSF). 
A

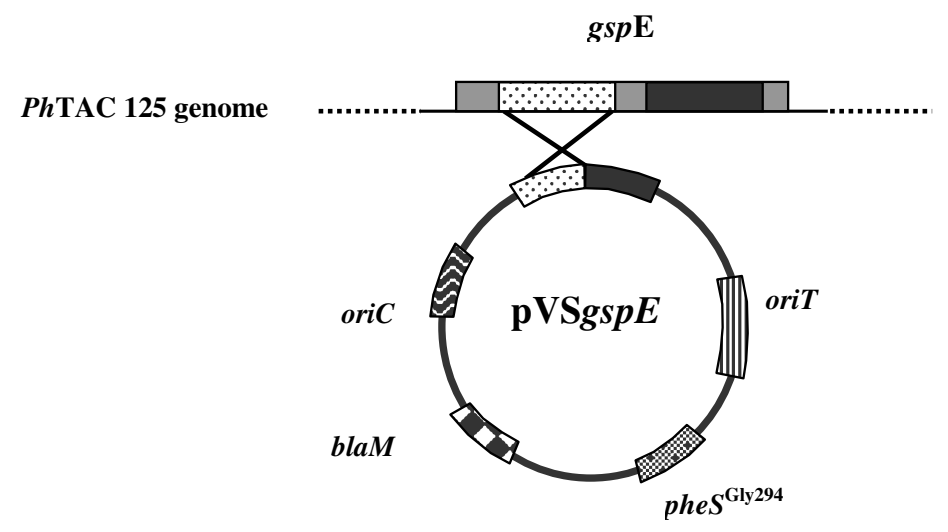

B
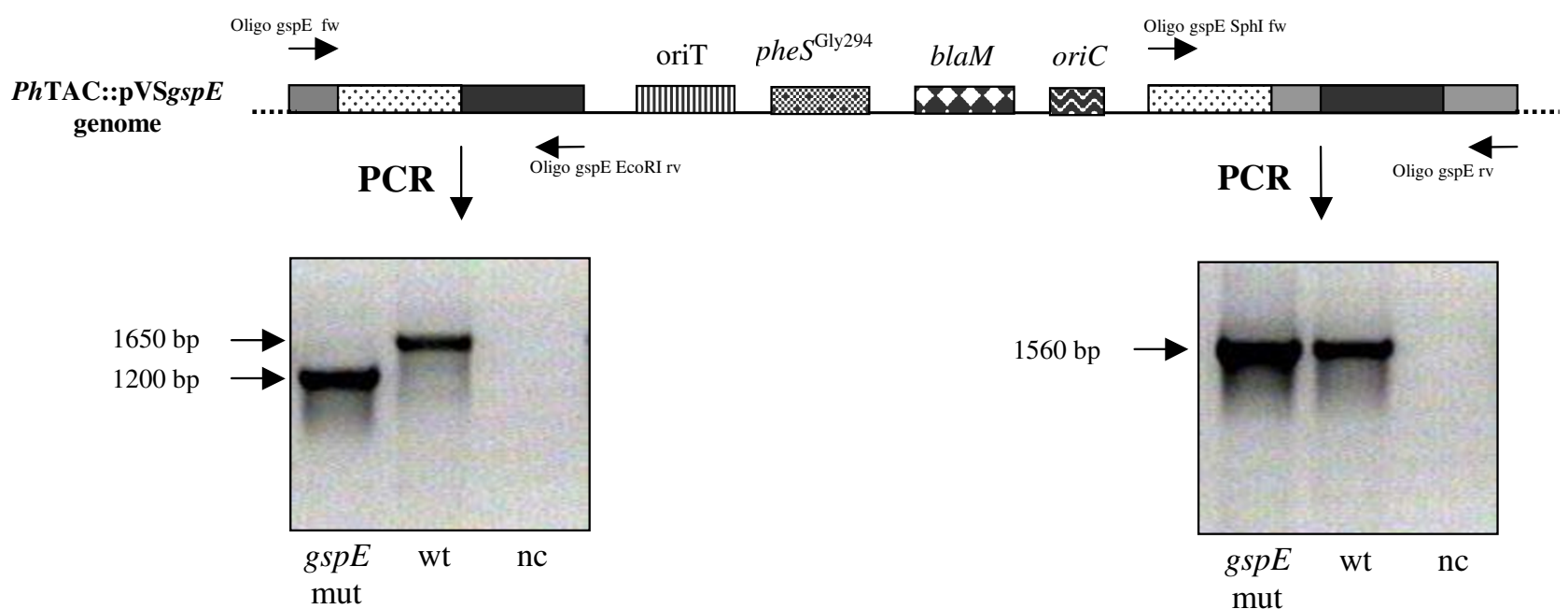

Figure 2

Schematic representation of pVS suicide vector (panel A) and genetic organization of $P$. haloplanktis TACI 25 gspE mutant (panel B). See text for details.

\section{Pseudoalteromonas haloplanktis TACI 25 genome} encodes a functional Type II protein secretion system Annotation of $P$. haloplanktis TAC125 genome sequence [15] revealed that the psychrophilic bacterium possesses only a canonical extra-cellular protein secretion pathway, i.e. the Type II secretion system (T2SS). The psychrophilic T2SS is located on the larger $P$. haloplanktis TAC125 chromosome and it is made up of twelve genes, from the gene PSHAa0231 to PSHAa0242. The gsp gene cluster is likely arranged in several independent transcriptional units, as previously observed in other Gram-negative bacteria $[19,20]$. Gene expression of P. haloplanktis TAC125 T2SS encoding cluster was evaluated by Reverse Transcriptase (RT)-PCR. Total RNA was extracted from cell samples collected at different growth phases, and was used to assess the transcription of $g s p E$, $g s p C$, and $g s p N$. All the tested $g s p$ genes resulted to be constitutively expressed in $P$. haloplanktis TAC125 cells (data not shown).

\section{Construction and genetic characterization of} Pseudoalteromonas haloplanktis TACI 25 gspE mutant strain

Functional inactivation of P. haloplanktis TAC125 T2SS system was achieved by insertional mutagenesis of $g s p E$ gene. This target was selected because it encodes an inner membrane-associated ATP-synthase, which has previously been reported to be essential for the T2SS functioning in other bacteria [21]. Insertional mutagenesis was obtained by using a suicide vector (pVS), suitably constructed for $P$. haloplanktis TAC125. As shown in Figure $2 \mathrm{~A}$, the pVS vector is characterized by the presence of: i) the pJB3-derived oriT [22], a DNA fragment responsible 
for the initiation of the conjugative transfer between an Escherichia coli S17-1 $\lambda$ pir strain (donor) and the psychrophilic cells (acceptor); ii) the E. coli blaM gene, encoding a mesophilic $\beta$-lactamase which is used as selection gene to isolate the first site-specific integration event; iii) pheSGly294, which encodes a mutated version of the E. coli $\alpha$ subunit of Phe-tRNA synthase [23], which renders bacteria sensitive to $p$-chlorophenylalanine. This phenylalanine analogue can be used as counterselective agent for the isolation of those strains in which a second recombination event occurred.

Two gspE gene fragments were amplified by PCR using specific oligonucleotides as primers. They correspond to two internal gene fragments and they are not adjacent (Figure 2A). The fragments were suitably digested and cloned into the $\mathrm{pVS}$ vector. The resulting vector ( $\mathrm{pVSgspE}$ ) was mobilized into $P$. haloplanktis TAC125, and clones in which a single recombination event occurred were selected on carbenicellin containing solid medium. Genomic DNA from some carbenicellin-resistant clones was extracted and subjected to different PCR analyses, to characterize the occurred genomic insertion. A positive clone was selected for further characterization, and its genome organization is reported in Figure 2B. P. haloplanktis TAC125::VSgspE mutant (hereafter called $P$. halo- planktis TAC125 gspE mutant) resulted to contain two copies of $g s p E$ gene, both carrying a specific deletion that was checked by sequencing the specific PCR products shown in Figure 2B. The first copy of $g s p E$ gene differs from the wild type one in lacking i) the region (450 bp long) between the two amplified fragments and ii) the $3^{\prime}$ encoding region (Figure 2B). A transcriptional analysis demonstrated that this $g s p E$ copy is transcribed (data not shown), but due to the large deletion, the resulting gene contains a translation frame shift leading to the production of an abnormal protein. The other gspE gene copy (Figure 2B) lacks of its promoter region and of the $5^{\prime}$ encoding portion, and therefore it resulted to be not transcribed (data not shown).

P. haloplanktis TAC125::VSgspE mutant was subjected to $p$ chlorophenylalanine treatment to select a clean deletion mutant. Although the counter-selection was carried out in several experimental conditions, all the selected clones resulted to be meroploid strains, containing both inserted and wild type alleles (data not shown).

\section{Pseudoalteromonas haloplanktis TACI 25 gspE mutant strain displays a reduced extra-cellular protease activity} Growth behaviour of $P$. haloplanktis TAC125 gspE mutant strain in standard conditions was determined, and com-
A

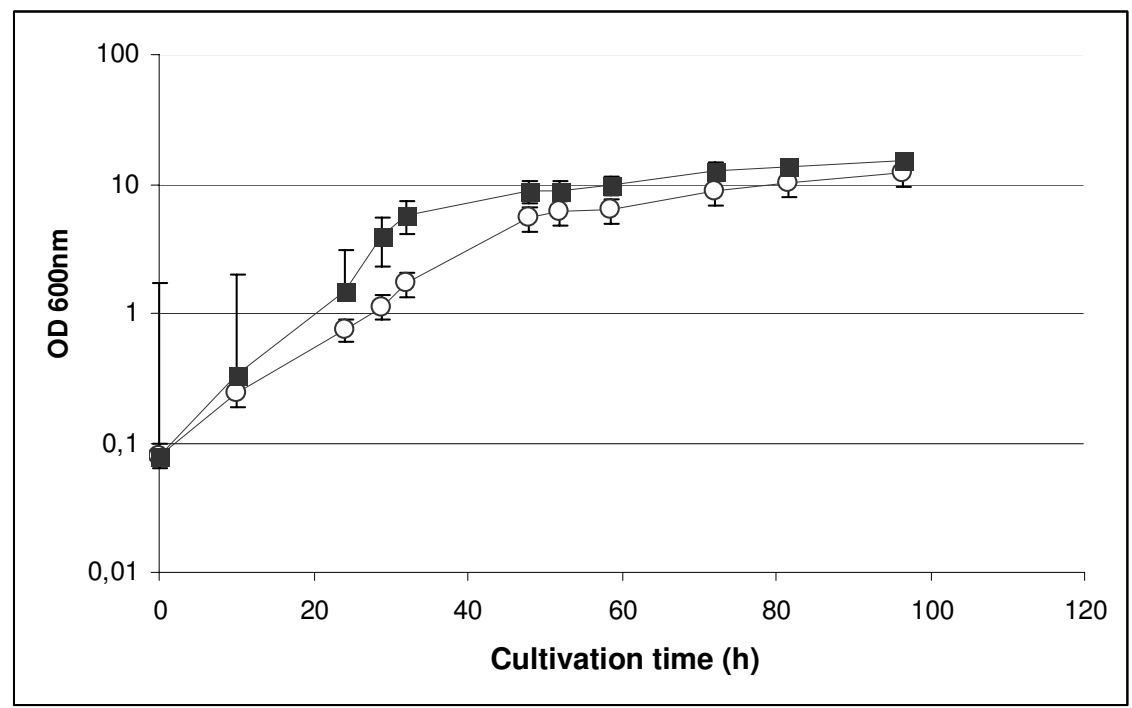

\section{Figure 3}

Comparison of $P$. haloplanktis TACI 25 wild type and gspE mutant growth kinetics and extra-cellular protein contents. Panel A: growth kinetics of $P$. haloplanktis TACI 25 wild type (open circle) and gspE mutant (solid square) in TYP medium at $4^{\circ} \mathrm{C}$. Panel B: I $2 \%$ SDS-PAGE analysis of tenfold concentrated culture supernatants of $P$. haloplanktis TACI 25 wild type and gspE mutant cells grown for 32 hours.
B

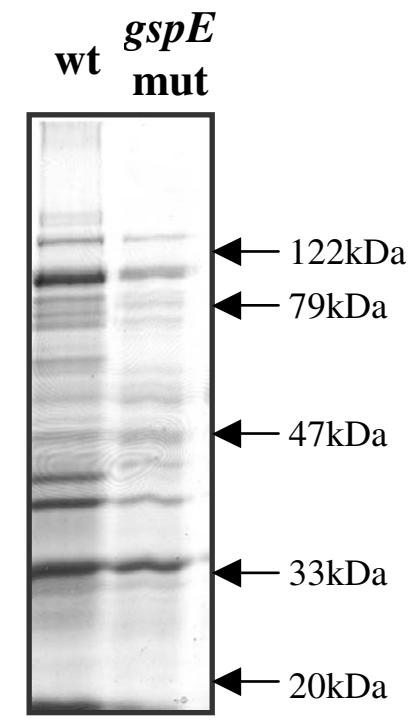


pared with the wild type one. As shown in Figure 3A, the gspE mutant strain grows faster and makes a higher cellular biomass than the wild type strain. Concentrated culture supernatants of $P$. haloplanktis TAC125 wild type and gspE mutant were analyzed by SDS-PAGE and results are shown in Figure 3B. The gspE mutant culture supernatant contains a reduced number of proteins compared to the wild type.

Extra-cellular protease secretion in P. haloplanktis TAC125 $g s p E$ mutant strain was investigated by gelatine zymography of concentrated culture supernatants. As shown in Figure $1 \mathrm{C}$, the samples contain a notably reduced number of proteolytic activities as compared to the protease content of wild type samples (Figure 1A) at the corresponding growth phases. Interestingly, the combined EDTA/PMSF treatment resulted in the almost complete inhibition of the extra-cellular proteases secreted by $g s p E$ mutant (Figure $1 \mathrm{D})$.

\section{Pseudoalteromonas haloplanktis TACI 25 gspE mutant strain is an improved host for the recombinant protein secretion at low temperature}

Secretion of psychrophilic $\alpha$-amylase in $P$. haloplanktis TAC125 gspE mutant cells was studied. The mutant strain was transformed with pFCamy $\Delta \mathrm{Ct}$, a psychrophilic vector previously constructed for the recombinant secretion of $\alpha$ amylase in the Antarctic bacterium [6]. The recombinant mutant strain was grown at $4^{\circ} \mathrm{C}$ till medium exponential phase and the $\alpha$-amylase secretion was evaluated by Western blotting analysis of cellular (Figure 4, lanes 3) and extra-cellular (Figure 4, lanes 4) protein samples. The

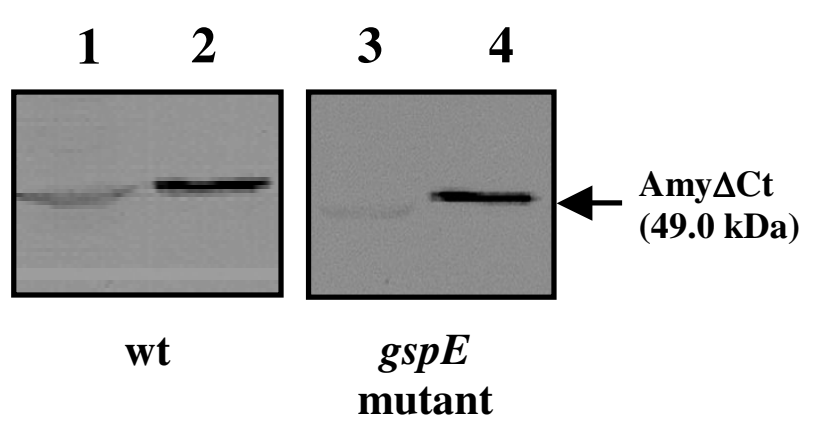

\section{Figure 4}

Psychrophilic $\alpha$-amylase secretion in $P$. haloplanktis TAC I 25 gspE mutant strain. Western blotting analysis of extra-cellular media (lane 4) and corresponding cellular extract (lane 3) of $P$. haloplanktis TACI 25 gspE mutant transformed with $\mathrm{pFCamy} \Delta \mathrm{Ct}$ plasmid. The western blotting analysis of extra-cellular media (lane 2) and corresponding cellular extract (lane I) of recombinant $P$. haloplanktis TACI25-(pFCamy $\Delta \mathrm{Ct}$ ) recombinant cells is shown as a control. results demonstrated that the $g s p E$ mutation does not affect secretion of the psychrophilic enzyme.

The secretion of the chimerical protein Amy $\Delta \mathrm{Ct}-\mathrm{BlaM}$, made up of the psychrophilic amylase fused to the mature $\beta$-lactamase [6], in P. haloplanktis TAC125 gspE mutant cells was investigated. P. haloplanktis TAC125 gspE mutant(pFCamy $\Delta$ Ct-blaM) recombinant cells were grown in TYP medium at $4{ }^{\circ} \mathrm{C}$ and samples were collected at different growth phases (early, and medium exponential phase). Culture supernatants were analysed by Western blotting analysis using anti- $\beta$-lactamase (anti-EcBla) and anti- $\alpha$ amylase (antiPh $\alpha$-Amy) polyclonal antisera, respectively. As shown in Figure $5 \mathrm{~B}$ lanes 3 and 4, both antisera detected a single product, with an apparent molecular weight of $80 \mathrm{kDa}$, corresponding to the Amy $\Delta \mathrm{Ct}$-BlaM chimerical protein. When a similar analysis was applied to wild type $P$. haloplanktis TAC125-(pFCamy $\Delta C t-b l a M)$ recombinant cells (Figure 5A lane 1 and 2), culture supernatants contained either the chimerical protein or its different proteolysis products, i.e. Amy $\Delta \mathrm{Ct}$, BlaM, and a chimera truncated form due to the action of host-encoded secreted proteases on the passenger protein.

\section{Discussion}

In a previous paper [6], we reported the establishing of a versatile gene-expression system for secretion of heterologous proteins in $P$. haloplanktis TAC125. The system uses the psychrophilic $\alpha$-amylase from $P$. haloplanktis TAB23 as secretion carrier, and allows an efficient extra-cellular addressing of recombinant proteins. However, we realized that the system efficiency was hampered by the presence of host-encoded extra-cellular proteolytic activities [6]. The presence of extra-cellular proteases represents a severe limit to the use of any genetic system for the recombinant protein secretion, since host-encoded proteases can affect the quality of the heterologous secreted products.

To enhance the recombinant product quality and stability, we focused our attention on the extra-cellular proteases produced by the psychrophilic $P$. haloplanktis TAC125 with the aim of developing a host mutant strain with reduced extra-cellular proteolytic activity.

By in gel activity assay, we demonstrated that several proteases are present in the culture medium of the psychrophilic bacterium (Figure 1A). The zymographies presented in Figure 1 did not allow us to define the exact number of extra-cellular proteases, due to the low resolution of this experimental technique. However, the inhibition assay allowed us to explore at least the diversity in the action mechanisms of the psychrophilic extra-cellular proteases. Indeed, metalloproteases, serine proteases and enzymes belonging to different classes were detected (Figure $1 \mathrm{~B})$. 
wt

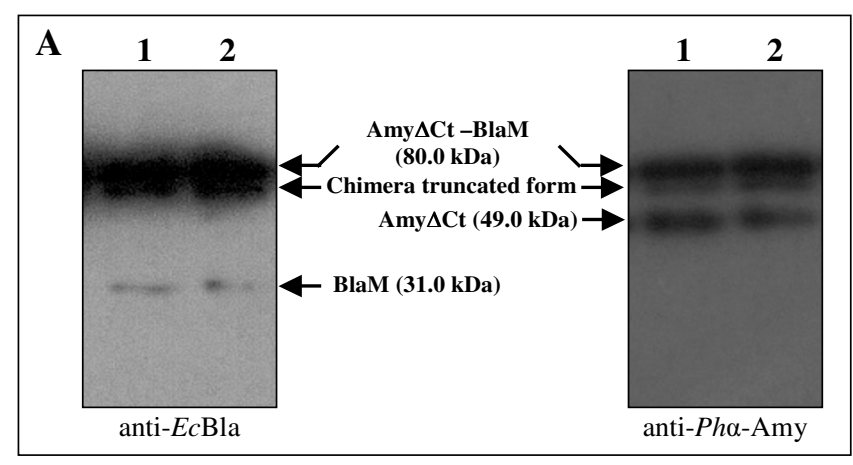

gspE mutant

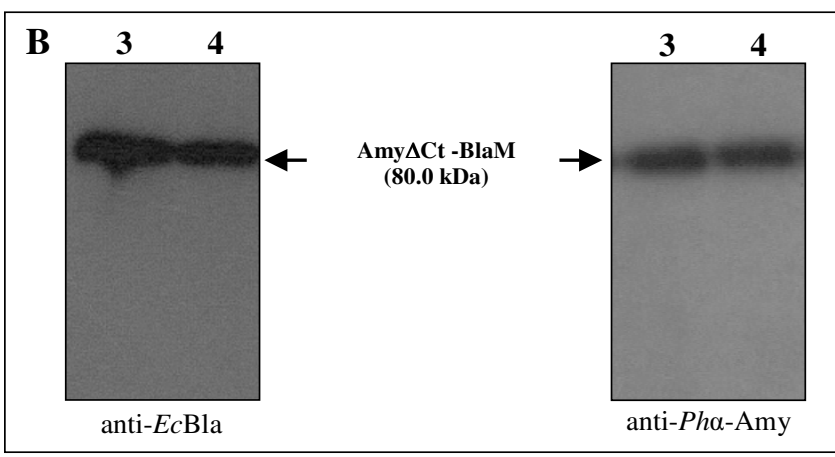

\section{Figure 5}

Amy $\Delta$ Ct-BlaM chimera secretion in P. haloplanktis TACI 25 wild type and gspE mutant. Panel A: Western blotting analyses of extra-cellular media of $P$. haloplanktis TACI 25(pFCamy $\Delta$ Ct-blaM) recombinant cells. Samples were collected during the early ( $24 \mathrm{~h}$ ) (lane I), and middle (32 h) (lane 2) exponential phase. Immunodetection was performed by chemioluminescence after probing proteins with anti- $\alpha$ amylase (anti-Ph $\alpha$-Amy) and anti- $\beta$-lactamase (anti-EcBla) polyclonal antisera. Panel B: Western blotting analyses of extra-cellular media of $P$. haloplanktis TACI 25 gspE mutant(pFCamy $\Delta$ Ct-blaM) recombinant cells. Samples were collected during the early ( $24 \mathrm{~h}$ ) (lane 3 ), and middle ( $32 \mathrm{~h}$ ) (lane 4) exponential phase. The immunodetections were performed by using anti- $\alpha$-amylase (anti-Ph $\alpha$-Amy) and anti- $\beta$ lactamase (anti-EcBla) polyclonal antisera.

Considering the multiplicity and heterogeneity of proteases detected in P. haloplanktis TAC125 culture medium, the systematic disruption of each extra-cellular protease encoding gene appears a time-consuming strategy. Therefore, we decided to inactivate the molecular machinery responsible for the extra-cellular targeting of proteases.

This aim was achieved combining some information deriving from the in silico analysis of $P$. haloplanktis TAC125 genome with several experimental evidences. In particular, from the accurate genome annotation, it was known that the psychrophilic bacterium possesses only one canonical secretion machinery (the T2SS pathway), while we have collected evidences indicating that the psychrophilic $\alpha$-amylase (the secretion carrier of our recombinant system) is likely secreted by another secretion apparatus not yet fully characterized (unpublished results from this laboratory). Thus, the functional inactivation of P. haloplanktis TAC125 T2SS seemed a feasible approach to generate a psychrophilic mutant strain possibly secreting a lower protease amount but still able to secrete the recombinant $\alpha$-amylase and its chimerical derivates.

T2SS (also called General Secretory Pathway) is a multicomponent machinery encoded by the gsp cluster and promoting secretion of Sec- and Tat-dependent exoenzymes in a two-step process $[16,19,20]$. Our results demonstrated that the P. haloplanktis TAC125 gsp gene cluster is actually transcribed and that the gsp genes expression seems to be constitutive over the bacterial growth (data not shown). Therefore it was necessary to use a genetic approach aimed at completely abolishing gsp-dependent secretion during all growth phases. gspE Gene $[17,18]$ encodes a specialized ATP-synthase whose inactivation resulted in the total loss of T2SS functionality in other Gram-negative bacteria [21,24,25]. Therefore the psychrophilic $g s p E$ gene was selected as target for inactivation by insertional mutagenesis.

The P. haloplanktis TAC125 gspE mutant strain was constructed by applying a typical gene targeting strategy, which makes use of a suitably constructed psychrophilic suicide vector (Figure $2 \mathrm{~A}$ ). As described in result section, this mutagenesis strategy allowed the creation of a $P$. haloplanktis TAC125 mutant strain characterized by the absence of GspE function.

A preliminary phenotypic analysis, carried out in standard growth conditions, demonstrated that $P$. haloplanktis TAC125 gspE mutant displays specific growth rate and biomass productivity higher than the wild type strain, thus surprisingly the mutation does not affect but improve the bacterium fitness (Figure 3A). This observed $g s p E$ mutant behaviour could be justified considering that gsp-dependent protein secretion is a high costly metabolic process, and its inhibition may represent an advantage to $g s p E$ mutant strain, at least in the tested growth condition.

The analysis of the extra-cellular protein content demonstrated that $g s p E$ mutant actually secretes lower amounts of proteins with respect to the wild type strain (Figure $3 \mathrm{~B}$ ). Proteins secreted by the gspE mutant are translocated by secretion pathways different from T2SS, such as the specialized machineries for type IV pili and curli components secretion [15] and the secretion apparatus responsible for 
the recombinant $\alpha$-amylase secretion (unpublished results from this laboratory).

Zymographic analysis revealed that the gspE mutant strain medium contains a notably reduced number of proteolytic activities (Figure 1C) with respect to wild type culture supernatants (Figure 1A). In contrast, knocking out of gspE gene did not impair for secretion of the cold-adapted amylase (Figure 4). In fact, P. haloplanktis TAC125 wild type and $g s p E$ mutant strains resulted to be indistinguishable in terms of cold-adapted $\alpha$-amylase production and secretion yields.

The gspE mutant was further tested for its ability to secrete a $\alpha$-amylase chimerical product, the Amy $\Delta \mathrm{Ct}-\mathrm{BlaM}$ chimera. When produced by wild type cells, the chimera components (i.e. the psychrophilic $\alpha$-amylase and the mesophilic $\beta$-lactamase) (Figure $5 \mathrm{~A}$, lanes 1 and 2 ) are partially separated due to the sub-stoichiometric proteolytic cleavage of the linker which connects the two proteins (see also [6]). This processing does not affect the catalytic activity of each component of the chimera [6]. The host-encoded secreted proteases are also responsible for further chimera degradation (see "chimera truncated form" in figure 5A, lanes 1 and 2) which accounts for the previously reported and undesired decrease of passenger activity [6]. On the contrary, due to the reduced number of extra-cellular proteases present in culture medium, Amy $\Delta$ Ct-BlaM chimera accumulates as a unique unprocessed form when produced in $P$. haloplanktis TAC125 gspE mutant cells (Figure 5B, lanes 3 and 4), thus resulting in an enhancement of chimera quality over the whole production process.

\section{Conclusion}

Combining the experimental evidences we collected on the $\alpha$-amylase secretion machinery in $P$. haloplanktis TAC125 with the careful in silico analysis of its genome, we designed an simple and successful experimental approach for the construction of an improved psychrophilic host for the cold $\alpha$-amylase-dependent recombinant secretion system. In fact, by a single gene disruption, involving the psychrophilic gspE gene, we developed a $P$. haloplanktis TAC125 mutant strain which secretes a significantly reduced extra-cellular protease activity while keeping its ability to secrete the recombinant psychrophilic $\alpha$-amylase as the wild type strain. The gspE mutant strain is also characterized by specific growth rate and biomass productivity higher than wild type strain, making it a truly improved host with a remarkable biotechnological potential in recombinant protein secretion at low temperature. Moreover, this work demonstrates that $P$. haloplanktis TAC125 is a versatile psychrophilic host for recombinant protein production since it that can be easily improved by a directed engineering approach. To the best of our knowl- edge, this is the first described example of a strain improvement strategy applied to an Antarctic bacterium.

\section{Methods}

\section{Strains and plasmids}

P. haloplanktis TAC125 was isolated from Antarctic sea water [15]. Escherichia coli DH5 $\alpha$ [26] was used as host for the gene cloning. E. coli strain S17-1( $\lambda$ pir) was used as donor in interspecific conjugation experiments [27].

\section{Growth conditions and analytical procedures}

P. haloplanktis TAC125 was grown in aerobic conditions at $4^{\circ} \mathrm{C}$ in TYP broth (16 gr/L yeast extract, $16 \mathrm{gr} / \mathrm{L}$ bacto tryptone, $10 \mathrm{gr} / \mathrm{L}$ marine mix) at $\mathrm{pH} 7.5$, supplemented with ampicillin $200 \mu \mathrm{g} / \mathrm{ml}$, chloramphenicol $25 \mu \mathrm{g} / \mathrm{ml}$, or cabenicellin $30 \mu \mathrm{g} / \mathrm{ml}$, when required. Antarctic bacteria transformation was achieved by intergeneric conjugation as previously reported [9].

E. coli cells were routinely grown in Terrific broth [28] at $37^{\circ} \mathrm{C}$. When required, antibiotics were added at the following concentrations in liquid cultures: $100 \mu \mathrm{g} / \mathrm{ml}$ of ampicillin, or cloramphenicol at $50 \mu \mathrm{g} / \mathrm{ml}$ final concentration. Genetic manipulations were carried out following standard procedures [28].

P. haloplanktis TAC125 DNA genomic purification was performed by ChargeSwitch gDNA Mini Bacteria Kit (Invitrogen).

Protein samples were analyzed by Polyacrylamide Gel Electrophoresis (Sodium Dodecyl Sulphate-PAGE) (12\% acrylamide, w/v) according to standard methods [28]. For immunoblotting, the proteins were transferred to a polyvinylidene difluoride membrane (Immobilon PSQ, Millipore). For immunodetection of proteins, $P$. haloplanktis TAB23 anti- $\alpha$-amylase [29] or anti- $\beta$-lactamase antisera were diluted in blocking buffer (phosphate buffer saline; $5 \%$ skimmed milk). Peroxidase conjugate anti-rabbit IgG (Sigma-Aldrich, USA) was used as secondary antibody. Proteins were detected by chemiluminescence's (Pierce, USA).

\section{Construction of suicide insertion vector pVSgspE}

pVS suicide vector was constructed by the insertion of the pJB3-derived oriT [22] and pheSGly294 gene [23] into the pGEM7Z vector. The oriT, responsible for the initiation of the conjugative transfer, was amplified on $\mathrm{pJB} 3$ vector by using Oligo oriTEcoRIfw and Oligo oriTSacIrv as primers (see Table 1), and was cloned into EcoRI and SacI sites of pGEM7Z (pGEM7Z-OriT). pheSGly294 gene, which encodes a mutated version of the E. coli $\alpha$ subunit of Phe-tRNA synthase, was amplified using pKSS [23] vector as template. The PCR reaction was carried out using the oligonucleotide pair PheSSNfw and PheSXrv, designed to 
introduce NdeI and $\mathrm{XbaI}$ restriction sites. The amplified DNA fragment was subjected to double NdeI/XbaI digestion and cloned into pPM13 plasmid [11] corresponding sites generating pPM13-pheSGly294 vector. The DNA fragment, containing P13 promoter and pheSGly294 gene, was recovered from pPM13-pheSGly294 vector by SmaI/EcoRI digestion, filled in and cloned into the pGEM7Z-oriT NaeI restriction site, resulting in the construction of the pVS vector.

Two DNA fragments of $P$. haloplanktis TAC125 gspE gene were amplified by PCR using bacterial genomic DNA as template. Two primer pairs were designed to amplify a 567 bp region at the $5^{\prime}$ end (Oligo gspESphIfw, Oligo gspESacIrv) and a $621 \mathrm{bp}$ region at the 3' (Oligo gspESacIfw, Oligo gspEEcoRIrv) end of the gspE gene. The amplified DNA fragments were digested by SphI/SacI and EcoRI/ SacI and cloned into the pVS SphI/EcoRI site to generate the $\mathrm{pVSgsp} E$ vector. The resulting vector was mobilized by intergeneric conjugation [9] into P. haloplanktis TAC125, and the cells were plated at $4{ }^{\circ} \mathrm{C}$ on TYP solid medium containing $30 \mu \mathrm{g} / \mathrm{ml}$ carbenicellin to select those clones in which a single recombination event occurred.

All PCR amplifications were performed in standard conditions [28]. The amplified fragments were cloned and their nucleotide sequences were checked to rule out the occurrence of any mutation during synthesis.

\section{Zymographic assay}

$P$. haloplanktis TAC125 wild type and gspE mutant strains were grown in standard conditions and culture samples were collected at different growth phases as reported in the text. Samples were centrifuged at $10000 \times g$ for $5 \mathrm{~min}$ at $4^{\circ} \mathrm{C}$ and the upper phase was collected for further analysis. The collected culture media were tenfold concentrated by Centricon (AMICON, exclusion size $5 \mathrm{kDa}$ ), and $12 \mu \mathrm{l}$ were loaded onto a non reducing SDS-PAGE containing gelatine $\left(1.5 \mathrm{mg} \mathrm{ml}^{-1}\right)$. After electrophoresis, gel was soaked twice with $2.5 \%$ Triton X-100 (v/v) solution for a total of $60 \mathrm{~min}$ to remove SDS. The gel was then incubated in a developing buffer $(50 \mathrm{mM}$ Tris- $\mathrm{HCl}, \mathrm{pH}$ 7.5, containing $5 \mathrm{mM} \mathrm{CaCl}_{2}$ ) for 12 or 18 (as indicated) hours at $15^{\circ} \mathrm{C}$, rinsed with water, and stained with Coomassie blue R250. Areas of gelatine digestion, corresponding to proteolytic activities, were visualized as unstained regions in the gel.

\section{Protease inhibition assay}

Tenfold concentrated culture supernatants of $P$. haloplanktis TAC125 wild type and $g s p E^{-}$mutant cells were incubated with no inhibitors, or $10 \mathrm{mM}$ EDTA, or $10 \mathrm{mM}$ PMSF, or a combination of EDTA and PMSF (both at 10 $\mathrm{mM}$ final concentration) at $15^{\circ} \mathrm{C}$ for 20 hours. The samples were then subjected to protease zymographic assay.

\section{Authors' contributions}

DDV and CC performed the experiments and helped to draft the manuscript. EP and MLT drafted the manuscript and designed and coordinated the study. All authors read and approved the manuscript

\section{Acknowledgements}

We are grateful to Dr. Gerard Michel (LISM/IBSM CNRS 3 I Chemin J. Aiguier I 3402 Marseille) for critical reading of the manuscript and very helpful discussions.

Table I: Plasmids and oligonucleotides used in this work

\begin{tabular}{|c|c|c|}
\hline Plasmids & & \\
\hline $\mathrm{pFC}$ & $\begin{array}{l}\text { Psychrophilic gene-expression vector, containing the T/R box, the promoter and termination region of the } P \text {. } \\
\text { haloplanktis TACI } 25 \text { aspC gene and the chloramphenicol resistance gene }\end{array}$ & [6] \\
\hline $\mathrm{pFCamy} \Delta \mathrm{Ct}$ & pFC containing a truncated version of the amy gene devoid of the C-terminal propeptide encoding portion & [6] \\
\hline pFCamy $\Delta$ Ct-blaM & $\mathrm{pFC}$ containing the $a m y \Delta \mathrm{Ct}-\mathrm{blaM}$ gene encoding the chimerical protein Amy-BlaM & [6] \\
\hline pVS & $\begin{array}{l}\text { PGEM7Z vector containing a conjugation transfer origin (oriT), the counter selectable marker pheS Gly } 294 \text { and the } \\
\text { ampicillin resistance gene }\end{array}$ & This work \\
\hline \multicolumn{3}{|l|}{ Oligonucleotides } \\
\hline Oligo oriTEcoRIfw & 5'-TTGAATTCTCGCACGATATACAGG-3' & \\
\hline Oligo oriTSaclrv & 5'-AAGAGCTCTTGAAGACGAAAGGG-3' & \\
\hline Oligo gspESphlfw & 5'-TTGCATGCATGCGCATCATCCGG-3' & \\
\hline Oligo gspESaclrv & 5'-AAGAGCTCCAATATCGAGCTTAGCC-3' & \\
\hline Oligo gspESaclfw & 5'-TTGAGCTCCTAAAGTAGGTATGACC-3' & \\
\hline Oligo gspEEcoRIrv & 5'-AAGAATTCGTACACGGGCTACAGCC-3' & \\
\hline Oligo gspESrv & 5'-AAGAGCTCCTTCACTGAGCATCG-3' & \\
\hline Oligo gspEfw & 5'-GCAATTTAAGCAGCGCGAAGATG-3' & \\
\hline Oligo gspErv & 5'-ATCTAGGGCACGGTATTCAAATGC-3' & \\
\hline PheSSNfw & 5'-TTGTCGACATATGTCACATCTCGCAGAAC-3' & \\
\hline PheSXrv & 5'-ССTCTAGAGAATTTCATAATCTATTCCTGCC-3' & \\
\hline
\end{tabular}


This work was supported by grants of Ministero dell'Università e della Ricerca Scientifica (Progetti di Rilevante Interesse Nazionale 2006) and of Programma Nazionale di Ricerche in Antartide 2004.

\section{References}

I. Cornelis P: Expressing genes in different Escherichia coli compartments. Curr Opin Biotechnol 2000, I I:450-4.

2. Makrides SC: Strategies for achieving high-level expression of genes in Escherichia coli. Microbiol Rev 1996, 60:5 12-38.

3. Mergulhaõ F, Monteiro G, Cabral J, Taipa M: Design of bacterial vector systems for the production of recombinant proteins in Escherichia coli. J Microb Biotechnol 2004, I 4: I- I4.

4. Mergulhaõ F, Monteiro G, Kelly A, Taipa M, Cabral J: Recombinant human proinsulin: a new approach in gene assembly and protein expression. J Microb Biotechnol 2000, 10:690-3.

5. Liao YD, Jeng JC, Wang CF, Wang SC, Chang ST: Removal of $\mathbf{N}$ terminal methionine from recombinant proteins by engineered $E$ coli methionine aminopeptidase. Protein Sci 2004, 13:1802-10.

6. Cusano AM, Parrilli E, Marino G, Tutino ML: A novel genetic system for recombinant protein secretion in the Antarctic Pseudoalteromonas haloplanktis TACI 25. Microb Cell Fact 2006, 1 4:5:40.

7. Georgiou G, Valax P: Expression of correctly folded proteins in Escherichia coli. Curr Opin Biotechnol 1996, 7:190-7.

8. Baneyx F: Recombinant protein expression in Escherichia coli. Curr Opin Biotechnol 1999, 10:4II-2I.

9. Duilio A, Tutino ML, Marino G: Recombinant protein production in Antarctic Gram-negative bacteria. Methods Mol Biol 2004, 267:225-237.

10. Vigentini I, Merico A, Tutino ML, Compagno C, Marino G: Optimization of recombinant Human Nerve Growth Factor production in the psychrophilic Pseudoalteromonas haloplanktis. J Biotechnol 2006, I 27( I): 141-50.

II. Papa R, Rippa V, Sannia G, Marino G, Duilio A: An effective cold inducible expression system developed in Pseudoalteromonas haloplanktis TACI 25. J Biotechnol 2007, I27:199-2I0.

12. Parrilli E, Duilio A, Tutino ML: Heterologous protein expression in psychrophilic hosts. In Psychrophiles: from Biodiversity to Biotechnolgy Edited by: Margesin R, Schinner F, Marx JC, Gerday C. SpringerVerlag Berlin Heidelberg; 2008:365-379.

13. Cusano AM, Parrilli E, Duilio A, Sannia G, Marino G, Tutino ML: Secretion of psychrophilic alpha-amylase deletion mutants in Pseudoalteromonas haloplanktis TACI 25. FEMS Microbiol Lett 2006, 258(I):67-7I.

14. Feller G, Lonhienne C, Deroanne C, Libioulle J, Van Beeumen J, Gerday C: Purification, characterization, and nucleotide sequence of the thermolabile alpha-amylase from the Antarctic psychrotroph Alteromonas haloplanctis A23. J Biol Chem |992, 267:52|7-522|.

15. Médigue C, Krin E, Pascal G, Barbe V, Bernsel A, Bertin PN, Cheung F, Cruveiller S, D'Amico S, Duilio A, Fang G, Feller G, Ho C, Mangenot S, Marino G, Nilsson J, Parrilli E, Rocha EPC, Rouy Z, Sekowska A, Tutino ML, Vallenet D, von Heijne G, Danchin A: Coping with cold: the genome of the versatile marine Antarctica bacterium Pseudoalteromonas haloplanktis TACI25. Genome Research 2005, 1 5: / 325-35.

16. Johnson TL, Abendroth J, Hol WG, Sandkvist M: Type II secretion: from structure to function. FEMS Microbiol Lett 2006, 255(2): 175-86. Review

17. Yamagata A, Tainer JA: Hexameric structures of the archaeal secretion ATPase GspE and implications for a universal secretion mechanism. EMBO J 2007, 26(3):878-90.

18. Chen Y, Shiue SJ, Huang CW, Chang JL, Chien YL, Hu NT, Chan NL: Structure and function of the XpsE N-terminal domain, an essential component of the Xanthomonas campestris type II secretion system. Biol Chem 2005, 280(5 I):42356-63.

19. Sandkvist M: Biology of type II secretion. Mol Microbiol 200I, 40(2):27I-83.

20. Sandkvist M: Type II secretion and pathogenesis. Infect Immun 200I, 69(6):3523-35.

21. Sandkvist M, Bagdasarian M, Howard SP, DiRita VJ: Interaction between the autokinase EpsE and EpsL in the cytoplasmic membrane is required for extra-cellular secretion in Vibrio cholerae. EMBO J 1995, I4(8): 1664-73.
22. Blatny JM, Brautaset T, Winther-Larsen HC, Haugan K, Valla S: Construction and use of a versatile set of broad-host-range cloning and expression vectors based on the RK2 replicon. Appl Environ Microbiol 1997, 63:370-9.

23. Kast P: pKSS-a second-generation general purpose cloning vector for efficient positive selection of recombinant clones. Gene 1994, 138:109-14.

24. Ball G, Chapon-Hervé V, Bleves S, Michel G, Bally M: Assembly of $\mathbf{X c p} R$ in the cytoplasmic membrane is required for extracellular protein secretion in Pseudomonas aeruginosa. J Bacteriol 1999, I 8 I (2):382-8.

25. Turner LR, Lara JC, Nunn DN, Lory S: Mutations in the consensus ATP-binding sites of XcpR and PilB eliminate extra-cellular protein secretion and pilus biogenesis in Pseudomonas aeruginosa. J Bacteriol 1993, 175(16):4962-9.

26. Hanahan D: Studies on transformation of Escherichia coli with plasmids. J Mol Biol 1983, 166:557-80.

27. Tascon RI, Rodriguez-Ferri EF, Gutierrez-Martin CB, Rodriguez-Barbosa I, Berche P, Va zquez-Boland JA: Transposon mutagenesis in Actinobacillus pleuropneumoniae with a $\mathrm{Tn} 10$ derivative. J Bacteriol 1993, 175:5717-22.

28. Sambrook J, Russell DW: Molecular Cloning in A Laboratory Manual 3rd edition. Cold Spring Harbor Laboratory, Cold Spring Harbor, NY; 2001.

29. Feller G, D'Amico S, Benotmane AM, Joly F, Van Beeumen J, Gerday $C$ : Characterization of the C-terminal propeptide involved in bacterial wall spanning of alpha-amylase from the psychrophile Alteromonas haloplanctis. J Biol Chem 1998, 273:12109-12115.
Publish with Bio Med Central and every scientist can read your work free of charge

"BioMed Central will be the most significant development for disseminating the results of biomedical research in our lifetime. "

Sir Paul Nurse, Cancer Research UK

Your research papers will be:

- available free of charge to the entire biomedical community

- peer reviewed and published immediately upon acceptance

- cited in PubMed and archived on PubMed Central

- yours - you keep the copyright

Submit your manuscript here:

http://www.biomedcentral.com/info/publishing_adv.asp
BioMedcentral 